\title{
SUBTHRESHOLD DOMAIN OF BISTABLE EQUILIBRIA FOR A MODEL OF HIV EPIDEMIOLOGY
}

\author{
B. D. CORBETT, S. M. MOGHADAS, and A. B. GUMEL
}

Received 20 September 2002

\begin{abstract}
A homogeneous-mixing population model for HIV transmission, which incorporates an anti-HIV preventive vaccine, is studied qualitatively. The local and global stability analysis of the associated equilibria of the model reveals that the model can have multiple stable equilibria simultaneously. The epidemiological consequence of this (bistability) phenomenon is that the disease may still persist in the community even when the classical requirement of the basic reproductive number of infection $\left(\mathscr{R}_{0}\right)$ being less than unity is satisfied. It is shown that under specific conditions, the community-wide eradication of HIV is feasible if $\mathscr{R}_{0}<\mathscr{R}_{*}$, where $\mathscr{R}_{*}$ is some threshold quantity less than unity. Furthermore, for the bistability case (which occurs when $\mathscr{R}_{*}<\mathscr{R}_{0}<1$ ), it is shown that HIV eradication is dependent on the initial sizes of the subpopulations of the model.
\end{abstract}

2000 Mathematics Subject Classification: 92B05, 37C75.

1. Introduction. It is well known that the basic reproductive number of infection $\left(\Re_{0}\right)$ being less than unity provides a necessary condition for community-wide eradication of an epidemic [1]. However, a number of studies have shown that this condition is not sufficient $[3,4,6,7,8,11,12,13,14]$. These studies have verified this fact by exploring the phenomenon of bistability, where multiple stable equilibria coexist, in some epidemic models. These models, in general, undergo backward bifurcations which are sufficient for the existence of stable endemic equilibria when $\mathscr{R}_{0}<1$ (see $[4,6,7,8,11,13]$ ). In other words, these studies have shown that a stable endemic equilibrium can coexist with a stable disease-free equilibrium. Thus, unlike in many classical disease transmission models (see, for instance, $[1,2,5,15,16]$ ), reducing $\mathscr{R}_{0}$ to values less than unity does not guarantee the community-wide eradication of an epidemic. This fact has important public health implications in the control or eradication of an epidemic.

The phenomenon of bistability has been observed in various types of epidemic models (see [13] for a general reference). For instance, Hadeler and Castillo-Chavez [10] studied the impact of the core group (the group of individuals who are sexually very active) on the existence of multiple infective steady-states in an epidemic model for some curable STDs. Feng et al. [6] considered an SEIT model for the transmission dynamics of TB with reinfection. 
They proved that a backward bifurcation occurs at $\mathscr{R}_{0}=1$ and that two endemic equilibria of the model coexist as long as $\mathscr{R}_{\mathcal{C}}<\mathscr{R}_{0}<1$, where $\mathscr{R}_{C}$ is a positive constant threshold. Kribs-Zaleta and Velasco-Hernández [14] presented an SVI vaccination model which exhibits a backward bifurcation under certain conditions. Gumel and Moghadas [9] proposed an SVIS vaccination model for the transmission dynamics of some curable diseases. Their study shows that although the model has no endemic equilibria under some conditions, changing the model parameters causes multiple endemic equilibria to occur when $\mathscr{R}_{0}<1$. Greenhalgh et al. [8] examined the impact of condom use on the dynamics of a multigroup SIR-type model of HIV/AIDS transmission amongst a male homosexual population. They showed, using numerical simulations, that their model has two endemic equilibria even when $\mathscr{R}_{0}<1$.

To the authors' knowledge, no rigorous qualitative study has been carried out to explore the effect of bistability on the transmission dynamics of HIV infection. Consequently, this study focuses on investigating the role of bistability in the spread and control of HIV within a homogeneous-mixing population. To achieve this objective, we consider a deterministic model of HIV transmission that incorporates anti-HIV preventive vaccine. Although there are numerous modes of HIV transmission (such as mother-to-child, needle-sharing by IV drug users, blood transfusion, etc.), our study focuses on HIV transmission via sexual means.

Our study leads to the determination of a certain threshold quantity $\mathscr{R}_{*}$ such that if $\mathscr{R}_{0}<\mathscr{R}_{*}$, then HIV will be eradicated from the community. This threshold quantity $\left(\mathscr{R}_{*}\right.$ ) gives a subthreshold domain of bistable equilibria of the model $\mathscr{R}_{*}<\mathscr{R}_{0}<1$, where the model has a stable endemic equilibrium coexisting with a stable disease-free equilibrium. Thus, the use of anti-HIV control measures that can reduce $\mathscr{R}_{0}$ below this threshold quantity (which leads to community-wide eradication of HIV) is of enormous public health importance.

The other feature of this study is the numerical estimation of the basins of attraction of the associated bistable equilibria of the model. These basins are separated by a stable manifold of an endemic equilibrium. Such estimate enables us to predict, for the bistability case, the persistence or eradication of HIV based on the initial sizes of the subpopulations of the model. Thus, controlling the initial sizes of the subpopulations can lead to the elimination of HIV infection in place of persistence.

This paper is organized as follows. The model is formulated in Section 2. In Section 3, the existence of the model equilibria is established under some specific conditions. Furthermore, by normalizing the model, the local and global stability of the associated equilibria are investigated. It is also shown that the model has no periodic orbits, homoclinic orbits, or polygons. The role of $\mathscr{R}_{0}$ on disease eradication are detailed in Section 4 . The threshold quantity $\mathscr{R}_{*}$ is also determined. Numerical simulations are reported in Section 5. 
2. Model formulation. The model monitors the temporal dynamics of three subpopulations, namely, the susceptible population $(S)$, the vaccinated population $(V)$, and the population of HIV-infected individuals (I). The total population is $N=S+V+I$. It should be mentioned that since the model under consideration monitors populations, it is henceforth assumed that all the associated model variables and parameters are nonnegative.

2.1. Susceptible population $(S)$. This population is generated following the recruitment of individuals at a rate of $\Pi$ per unit time. Recruitment is the inflow of people (either by birth or immigration) into a community. Since this study considers only sexual mode of HIV transmission, recruitment is defined in terms of the number of sexually active individuals admitted into the community per unit time. Furthermore, our model categorizes all individuals recruited into the community as susceptible. The population of susceptible individuals diminishes, following the acquisition of HIV infection which arises following contacts between a susceptible $(S)$ and the infectious fraction $(I / N)$ with a transmission probability $\beta_{1}$. The parameter $c$ represents the number of contact partners per unit time. This population is further diminished by the administration of anti-HIV preventive vaccine at a rate $\xi$ and by natural death at a rate $\mu$. This gives

$$
\frac{d S}{d t}=\Pi-\frac{c \beta_{1} S I}{N}-\xi S-\mu S
$$

2.2. Vaccinated population $(V)$. This population is generated by the vaccination of susceptibles at a rate $\xi$. It is diminished by HIV infection with a transmission probability $\beta_{2}$ and natural death at a rate $\mu$. It is assumed that the anti-HIV preventive vaccine reduces (but does not eliminate) the risk of HIV infection. Thus $\beta_{2} \leq \beta_{1}$. This can be summarized in the following equation:

$$
\frac{d V}{d t}=\xi S-\frac{c \beta_{2} V I}{N}-\mu V
$$

2.3. HIV-infected population ( $I$ ). This population is generated following the HIV infection of susceptible and vaccinated individuals. It diminishes by natural death at a rate $\mu$ and by progression to full-blown AIDS at a rate $\tau$. It is assumed that individuals with full-blown AIDS do not contribute to the spread of HIV infection. This gives

$$
\frac{d I}{d t}=\frac{c \beta_{1} S I}{N}+\frac{c \beta_{2} V I}{N}-(\mu+\tau) I .
$$




\section{Stability analysis}

3.1. Disease-free equilibrium. In the absence of HIV infection (i.e., $I=0$ ), the model, given by (2.1), (2.2), and (2.3), has a unique disease-free equilibrium

$$
E_{0}=\left(\frac{\Pi}{\mu+\xi}, \frac{\xi \Pi}{\mu(\mu+\xi)}, 0\right)
$$

To establish the local stability of $E_{0}$, the associated Jacobian of the model is evaluated at $E_{0}$. This gives

$$
\left(\begin{array}{ccc}
-(\mu+\xi) & 0 & -\frac{c \beta_{1} \mu}{\mu+\xi} \\
\xi & -\mu & -\frac{c \beta_{2}}{\mu+\xi} \\
0 & 0 & \frac{c \beta_{1} \mu}{\mu+\xi}+\frac{c \beta_{2} \xi}{\mu+\xi}-(\mu+\tau)
\end{array}\right)
$$

with eigenvalues

$$
\lambda_{1}=-(\mu+\xi), \quad \lambda_{2}=-\mu, \quad \lambda_{3}=\frac{c\left(\beta_{1} \mu+\beta_{2} \xi\right)}{\mu+\xi}-(\mu+\tau) .
$$

Since all the model parameters are assumed to be nonnegative, it follows that $\lambda_{1}$ and $\lambda_{2}$ are both negative. Thus, the stability of $E_{0}$ solely depends on the sign of $\lambda_{3}$. By defining

$$
\mathscr{R}_{0}=\frac{c\left(\beta_{1} \mu+\beta_{2} \xi\right)}{(\mu+\xi)(\mu+\tau)}
$$

it can be seen that $\lambda_{3}<0$ if and only if $\mathscr{R}_{0}<1$. Hence, we have established the following lemma.

LEMMA 3.1. The disease-free equilibrium $\left(E_{0}\right)$ is locally asymptotically stable if $\mathscr{R}_{0}<1$ and unstable if $\mathscr{R}_{0}>1$.

The quantity $\Re_{0}$, defined in (3.4), is the basic reproductive number of infection [1]. Lemma 3.1 shows that community-wide eradication of HIV is feasible provided that the initial sizes of the model subpopulations, namely, $S, V$, and $I$, are in the basin of attraction of $E_{0}$. However, if $E_{0}$ is globally asymptotically stable (see [2, 5, 15, 16]), then HIV will be eradicated from the community irrespective of the initial sizes of the subpopulations. The global stability of $E_{0}$ will be discussed in Section 3.3.

\subsection{Endemic equilibrium}

3.2.1. Existence of endemic equilibria. The endemic equilibria of the model (if they exist) correspond to the case where HIV infection persists $(I \neq 0)$. Since 
these equilibria cannot be clearly expressed in a closed form, we will investigate their existence under some specific conditions. To do this, we first define

$$
G(t)=\frac{c \beta_{1} I(t)}{N(t)}
$$

to be the force of infection (the rate of acquisition of new infected individuals per year [17]). It then follows that, at a steady state, (2.1) and (2.2) can be rewritten as

$$
S^{*}=\frac{\Pi}{\mu+\xi+G^{*}}, \quad V^{*}=\frac{\xi \Pi}{\left(\mu+\xi+G^{*}\right)\left(\mu+\left(\beta_{2} / \beta_{1}\right) G^{*}\right)} .
$$

Furthermore, using (3.5) in (2.3) gives (at equilibrium)

$$
I^{*}=\left(\frac{1}{\mu+\tau}\right)\left(\frac{\Pi G^{*}}{\mu+\xi+G^{*}}+\frac{\xi \beta_{2} \Pi G^{*}}{\beta_{1}\left(\mu+\xi+G^{*}\right)\left(\mu+\left(\beta_{2} / \beta_{1}\right) G^{*}\right)}\right) .
$$

Substituting $I^{*}$ from (3.7) into (3.5) and noting $N^{*}=S^{*}+V^{*}+I^{*}$ gives

$$
G^{*}=\frac{c\left(\mu \beta_{1}+\beta_{2} G^{*}+\xi \beta_{2}\right) G^{*}}{(\mu+\tau)\left(\mu+\left(\beta_{2} / \beta_{1}\right) G^{*}\right)+\xi(\mu+\tau)+\left(\mu+\left(\beta_{2} / \beta_{1}\right) G^{*}+\xi\left(\beta_{2} / \beta_{1}\right)\right) G^{*}} .
$$

By solving (3.8), the positive (endemic) equilibria of the model can be obtained using the expressions in (3.5) and (3.6). Clearly, $G^{*}=0$ is a fixed point of (3.8). Furthermore, this fixed point gives the disease-free equilibrium $E_{0}$ of the model (since (3.6) and (3.7) reduce to $S^{*}=\Pi /(\mu+\xi), V^{*}=\xi \Pi / \mu(\mu+\xi)$, and $I^{*}=0$ when $G^{*}=0$ ).

Suppose now that $G^{*} \neq 0$. In this case, (3.8) becomes

$$
\begin{gathered}
\beta_{2}\left(G^{*}\right)^{2}+\left[\mu \beta_{1}+\xi \beta_{2}+\beta_{2}(\mu+\tau+d)-c \beta_{1} \beta_{2}\right] G^{*} \\
+\beta_{1}\left[(\mu+\xi)(\mu+\tau)-c\left(\mu \beta_{1}+\xi \beta_{2}\right)\right]=0 .
\end{gathered}
$$

The endemic equilibria of the model can then be obtained by substituting the solutions of (3.9) into (3.6) and (3.7). In order to discuss the possible solutions of (3.9), we define

$$
A=(\mu+\xi)(\mu+\tau)-c\left(\mu \beta_{1}+\xi \beta_{2}\right)
$$

and consider the following cases.

CASE $1(A<0)$. In this case, (3.9) has real roots with opposite signs. Let $G_{+}^{*}$ denote the positive real root of (3.9). Thus, a unique positive endemic equilibrium of the model can then be obtained by substituting $G_{+}^{*}$ into the expressions of (3.6) and (3.7). 
CASE $2(A=0)$. This assumption reduces (3.9) to

$$
\left[\beta_{2} G^{*}+\mu \beta_{1}+\xi \beta_{2}+\beta_{2}(\mu+\tau)-c \beta_{1} \beta_{2}\right] G^{*}=0
$$

It is clear, in this case, that the root $G^{*}=0$ of (3.11) gives the disease-free equilibrium $E_{0}$. Let

$$
B=\mu \beta_{1}+\xi \beta_{2}+\beta_{2}(\mu+\tau)-c \beta_{1} \beta_{2}
$$

If $B<0$, then $G^{*}=-B / \beta_{2}$ is the unique positive root of (3.9) which corresponds to a unique endemic equilibrium of the model (obtained by substituting $G^{*}$ into the expressions of (3.6) and (3.7)). If $B \geq 0$, then (3.11) has no positive root. Hence, the model has no endemic equilibrium if $B \geq 0$.

CASE $3(A>0)$. Here, we consider the following three possibilities.

(a) Suppose $B^{2}-4 \beta_{1} \beta_{2} A>0$.

(i) If $B>0$, then the roots of (3.9) are both real and negative. Hence, the model has no endemic equilibrium.

(ii) If $B<0$, then (3.9) has two positive real roots. Thus, the model has two endemic equilibrium.

(iii) If $B=0$, then (3.9) has two complex roots and, in this case, no endemic equilibrium of the model exists.

(b) Suppose $B^{2}-4 \beta_{1} \beta_{2} A<0$. Under this assumption, (3.9) has no real roots. Thus, the model has no endemic equilibrium.

(c) Suppose $B^{2}-4 \beta_{1} \beta_{2} A=0$. This implies that (3.9) has a unique positive real root given by $G^{*}=-B / 2 \beta_{2}$ if $B<0$ and no positive root if $B \geq 0$. Thus, for $B^{2}-4 \beta_{1} \beta_{2} A=0$, the model has a unique endemic equilibrium if $B<0$ and no endemic equilibrium if $B \geq 0$.

Noting that $A=(\mu+\xi)(\mu+\tau)\left(1-\mathscr{R}_{0}\right)$, the above results can be summarized in Theorem 3.2.

THEOREM 3.2. (i) If $\mathscr{R}_{0}>1$, then the model has a unique endemic equilibrium.

(ii) If $\mathscr{R}_{0}=1$, then the model has a unique endemic equilibrium if $B<0$ and no endemic equilibrium if $B \geq 0$.

(iii) If $\mathscr{R}_{0}<1$ and $B^{2}-4 \beta_{1} \beta_{2} A>0$, then the model has two endemic equilibria if $B<0$ and no endemic equilibrium if $B \geq 0$.

(iv) If $\mathscr{R}_{0}<1$ and $B^{2}-4 \beta_{1} \beta_{2} A<0$, then no endemic equilibrium of the model exists.

(v) If $\mathscr{R}_{0}<1$ and $B^{2}-4 \beta_{1} \beta_{2} A=0$, then the model has a unique endemic equilibrium if $B<0$ and no endemic equilibrium if $B \geq 0$.

3.2.2. Nonexistence of periodic orbits. Using the results of the existence of the endemic equilibria, we will discuss their stability based on some qualitative properties of the model. To do this, the model represented by (2.1), (2.2), and 
(2.3) is normalized using the following change of variables and parameters:

$$
\begin{array}{r}
S_{1}=\frac{\mu}{\Pi} S, \quad V_{1}=\frac{\mu}{\Pi} V, \quad I_{1}=\frac{\mu}{\Pi} I, \\
\tilde{t}=\mu t, \quad \tilde{\beta}_{1}=\frac{c \beta_{1}}{\mu}, \quad \tilde{\beta}_{2}=\frac{c \beta_{2}}{\mu}, \quad \tilde{\xi}=\frac{\xi}{\mu}, \quad \tilde{\tau}=\frac{\tau}{\mu} .
\end{array}
$$

Thus, the normalized model has the form

$$
\begin{aligned}
\frac{d S_{1}}{d \tilde{t}} & =1-\frac{\tilde{\beta}_{1} S_{1} I_{1}}{N_{1}}-\tilde{\xi} S_{1}-S_{1}, \\
\frac{d V_{1}}{d \tilde{t}} & =\tilde{\xi} S_{1}-\frac{\tilde{\beta}_{2} V_{1} I_{1}}{N_{1}}-V_{1}, \\
\frac{d I_{1}}{d \tilde{t}} & =\frac{\tilde{\beta}_{1} S_{1} I_{1}}{N_{1}}+\frac{\tilde{\beta}_{2} V_{1} I_{1}}{N_{1}}-(1+\tilde{\tau}) I_{1},
\end{aligned}
$$

where $N_{1}=S_{1}+V_{1}+I_{1}$. Clearly, this normalized model has an equilibrium solution $e_{0}=(1 /(1+\tilde{\xi}), \tilde{\xi} /(1+\tilde{\xi}), 0)$ which corresponds to the disease-free equilibrium $E_{0}$ of the original model. It can be seen, by adding (3.15), (3.16), and (3.17), that

$$
\frac{d N_{1}}{d \tilde{t}}=1-N_{1}-\tilde{\tau} I_{1}
$$

Consequently, in the absence of HIV infection $\left(I_{1}=0\right)$, the total population size of the normalized model is $N_{1}=1$ (as $t \rightarrow \infty$ ). Since the spread of HIV infection within the community is expected to reduce $N_{1}$ (due to disease-induced death), we study the normalized model in the following feasible region:

$$
\mathscr{D}=\left\{\left(S_{1}, V_{1}, I_{1}\right): S_{1}, V_{1}, I_{1} \geq 0, S_{1}+V_{1}+I_{1} \leq 1\right\} .
$$

It follows from (3.18) that if $N_{1}>1$, then $d N_{1} / d \tilde{t}<0$. Hence, $\mathscr{D}$ is a positively invariant region for the normalized model. Furthermore, since $d N_{1} / d \tilde{t}<0$ when $S_{1}+V_{1}+(1+\tilde{\tau}) I_{1}>1$ and $d N_{1} / d \tilde{t}>0$ when $S_{1}+V_{1}+(1+\tilde{\tau}) I_{1}<1$, then

$$
\mathscr{D}^{*}=\left\{\left(S_{1}, V_{1}, I_{1}\right) \in \mathscr{D}: S_{1}+V_{1}+(1+\tilde{\tau}) I_{1}=1\right\}
$$

is also a positively invariant region for the normalized model. This implies that every solution of (3.15), (3.16), and (3.17) with an initial condition in $\mathscr{D}$ tends toward $\mathscr{D}^{*}$ as $t \rightarrow \infty$ and every solution with an initial condition in $\mathscr{D}^{*}$ remains there for $\tilde{t}>0$. Therefore, the $\omega$-limit sets of (3.15), (3.16), and (3.17) are contained in $\mathscr{D}^{*}$.

Here we will show, using [2, Lemma 3.1], the nonexistence of certain types of solutions such as periodic orbits, homoclinic orbits, or polygons associated with the normalized model.

THEOREM 3.3. The normalized model (3.15), (3.16), and (3.17) has no periodic orbits, homoclinic orbits, or polygons in the interior of $\mathfrak{D}^{*}$. 
Proof. Let $f_{1}, f_{2}$, and $f_{3}$ denote the right-hand sides of (3.15), (3.16), and (3.17), respectively. The relation $S_{1}+V_{1}+(1+\tilde{\tau}) I_{1}=1$ is used to obtain $f_{j}\left(V_{1}, I_{1}\right)$, $f_{k}\left(S_{1}, I_{1}\right)$, and $f_{l}\left(S_{1}, V_{1}\right)$ for $j=2,3, k=1,3$, and $l=1$, 2. Define $\mathbf{G}=g_{1}+g_{2}+g_{3}$ as a vector field with

$$
\begin{aligned}
& g_{1}\left(V_{1}, I_{1}\right)=\left[0, \frac{-f_{3}\left(V_{1}, I_{1}\right)}{V_{1} I_{1}}, \frac{f_{2}\left(V_{1}, I_{1}\right)}{V_{1} I_{1}}\right], \\
& g_{2}\left(S_{1}, I_{1}\right)=\left[\frac{f_{3}\left(S_{1}, I_{1}\right)}{S_{1} I_{1}}, 0, \frac{-f_{1}\left(S_{1}, I_{1}\right)}{S_{1} I_{1}}\right], \\
& g_{3}\left(S_{1}, V_{1}\right)=\left[\frac{-f_{2}\left(S_{1}, V_{1}\right)}{S_{1} V_{1}}, \frac{f_{1}\left(S_{1}, V_{1}\right)}{S_{1} V_{1}}, 0\right] .
\end{aligned}
$$

Clearly, $\mathbf{G} \cdot \mathbf{F}=0$ in the interior of $\mathscr{D}^{*}$, where $\mathbf{F}=\left(f_{1}, f_{2}, f_{3}\right)$. Using the normal vector $\mathbf{n}=(1,1,1+\tilde{\tau})$ to $\mathscr{D}^{*}$, it can be shown, after some tedious manipulations, that

$$
(\operatorname{CurlG}) \cdot(1,1,1+\tilde{\tau})=-\left(\frac{1-S_{1}}{S_{1}^{2} V_{1} I_{1}}+\frac{\tilde{\xi}}{V_{1}^{2} I_{1}}\right)<0 .
$$

Thus, it follows from [2, Lemma 3.1] that the normalized model (3.15), (3.16), and (3.17) has no periodic orbits, homoclinic orbits, or polygons.

As an immediate consequence of the above theorem, it can be seen that since $\mathscr{D}^{*}$ is a bounded-invariant set, it follows from the Poincaré-Bendixson theorem in two-dimensional simplex $\mathscr{D}^{*}$ that the $\omega$-limit set of every solution of the normalized model is an equilibrium point (see also [18]).

3.3. Stability analysis of the normalized model. It should be mentioned that since the infected population $I(t)$ is changing in time (except at equilibria), (3.18) shows that the size of the total population is not constant. Thus, the normalized model (3.15), (3.16), and (3.17) (and, consequently, the original model) cannot be reduced to a two-dimensional model (by eliminating one of the model variables). Here, we will discuss the stability of the equilibria of the normalized model in $\mathscr{D}^{*}$ by taking advantage of Theorems 3.2 and 3.3 as follows.

First of all, note that the expressions $A$ and $B$ (defined in (3.10) and (3.12)) can be rewritten in terms of the new parameters defined in (3.14). This gives

$$
\begin{aligned}
& \tilde{A}=\left[(1+\tilde{\xi})(1+\tilde{\tau})-\left(\tilde{\beta}_{1}+\tilde{\xi} \tilde{\beta}_{2}\right)\right] \mu^{2}, \\
& \tilde{B}=\left[\tilde{\beta}_{1}+\tilde{\xi} \tilde{\beta}_{2}+\tilde{\beta}_{2}(1+\tilde{\tau})-\tilde{\beta}_{1} \tilde{\beta}_{2}\right] \frac{\mu^{2}}{c} .
\end{aligned}
$$

Thus, we have the following result on the existence of the equilibria of the normalized model.

COROLLARY 3.4. (i) If $\tilde{A}<0$, then the normalized model has a unique endemic equilibrium in the interior of $\mathscr{D}^{*}$. 
(ii) If $\tilde{A}=0$, then the normalized model has a unique endemic equilibrium if $\tilde{B}<0$ and no endemic equilibrium if $\tilde{B} \geq 0$ in the interior of $\mathscr{D}^{*}$.

(iii) If $\tilde{A}>0$ and $\tilde{B}^{2}-4\left(\mu^{2} / c^{2}\right) \tilde{\beta}_{1} \tilde{\beta}_{2} \tilde{A}>0$, then the normalized model has two endemic equilibria if $\tilde{B}<0$ and no endemic equilibrium if $\tilde{B} \geq 0$ in the interior of $\mathscr{D}^{*}$.

(iv) If $\tilde{A}>0$ and $\tilde{B}^{2}-4\left(\mu^{2} / c^{2}\right) \tilde{\beta}_{1} \tilde{\beta}_{2} \tilde{A}<0$, then no endemic equilibrium of the normalized model exists in the interior of $\mathscr{D}^{*}$.

(v) If $\tilde{A}>0$ and $\tilde{B}^{2}-4\left(\mu^{2} / c^{2}\right) \tilde{\beta}_{1} \tilde{\beta}_{2} \tilde{A}=0$, then the normalized model has a unique endemic equilibrium if $\tilde{B}<0$ and no endemic equilibrium if $\tilde{B} \geq 0$ in the interior of $\mathscr{D}^{*}$.

It should be noted that, for the normalized model, the basic reproductive number $\mathscr{R}_{0}$ reduces to

$$
\tilde{\mathscr{R}}_{0}=\frac{\tilde{\beta}_{1}+\tilde{\xi} \tilde{\beta}_{2}}{(1+\tilde{\xi})(1+\tilde{\tau})} .
$$

It is easy to check that the disease-free equilibrium of the normalized model $e_{0}$ is locally asymptotically stable if $\tilde{\mathscr{R}}_{0}<1$ and unstable if $\tilde{\mathscr{R}}_{0}>1$. Furthermore, we have the following result.

THEOREM 3.5. The equilibrium $e_{0}$ of the normalized model is globally asymptotically stable if one of the following statements holds:

(i) $\tilde{\mathscr{R}}_{0} \leq 1$ and $\tilde{B} \geq 0$;

(ii) $\tilde{\mathscr{R}}_{0}<1$ and $\tilde{B}^{2}-4\left(\mu^{2} / c^{2}\right) \tilde{\beta}_{1} \tilde{\beta}_{2} \tilde{A}<0$.

Proof. We first note that $\tilde{\mathscr{R}}_{0} \leq 1$ if and only if $\tilde{A} \geq 0$. It follows from Corollary 3.4 that, in either of cases (i) and (ii), the normalized model has no endemic equilibrium in the interior of $\mathscr{D}^{*}$. Thus, $e_{0}$ is the only equilibrium point of the normalized model in $\mathscr{D}^{*}$. Since $\mathscr{D}^{*}$ is a bounded-positively invariant set and the model has no periodic orbit in the interior of $\mathscr{D}^{*}$ (by Theorem 3.3), the Poincaré-Bendixson theorem implies that the $\omega$-limit set of every solution must be the equilibrium point $e_{0}$. Consequently, $e_{0}$ is globally asymptotically stable.

For the stability of the unique endemic equilibrium of the normalized model, we offer the following theorem.

THEOREM 3.6. The normalized model has a unique endemic equilibrium in $\mathscr{D}^{*}$ which is globally asymptotically stable if one of the following statements holds: (i) $\tilde{\mathscr{R}}_{0}>1$; (ii) $\tilde{\mathscr{R}}_{0}=1$ and $\tilde{B}<0$.

Proof. It follows from Corollary 3.4 that the normalized model has a unique endemic equilibrium in the interior of $\mathscr{D}^{*}$ if one of the above cases ((i) or (ii)) holds. Since $\tilde{\mathscr{R}}_{0} \geq 1$, the equilibrium $e_{0}$ is unstable with a two-dimensional stable manifold and a one-dimensional unstable manifold. The stable manifold of $e_{0}$ is located in the $\left(S_{1}, V_{1}\right)$-plane. Thus, $e_{0}$ only attracts the region 
$\mathscr{D}_{0}=\left\{\left(S_{1}, V_{1}, 0\right): S_{1}+V_{1}=1\right\} \subset \mathscr{D}^{*}$. Since the model has no periodic orbits in the interior of $\mathscr{D}^{*}$, it follows from Theorem 3.3 and the Poincaré-Bendixson theorem that the $\omega$-limit set of every solution in the interior of $\mathscr{D}^{*}$ must be the unique endemic equilibrium. Thus, this endemic equilibrium is globally asymptotically stable in $\mathscr{D}^{*} \backslash \mathscr{D}_{0}$.

REMARK 3.7. It is clear that $e_{0}$ is locally asymptotically stable if $\tilde{A}>0\left(\tilde{\mathscr{R}}_{0}<\right.$ 1). The authors have tried to establish the stability of the endemic equilibrium whenever $\tilde{A}>0$ and $\tilde{B}^{2}-4\left(\mu^{2} / c^{2}\right) \tilde{\beta}_{1} \tilde{\beta}_{2} \tilde{A}=0$ (see Corollary $3.4(\mathrm{v})$ ) but without any success.

We now continue our analysis for the case where the normalized model has two endemic equilibria (namely, $e_{1}$ and $e_{2}$ ) where the following three conditions are satisfied (see Corollary 3.4(iii)):

$$
\tilde{A}>0, \quad \tilde{B}^{2}-4 \frac{\mu^{2}}{c^{2}} \tilde{\beta}_{1} \tilde{\beta}_{2} \tilde{A}>0, \quad \tilde{B}<0 .
$$

Here, we will show that these two endemic equilibria cannot be repellers (in $\mathscr{D}^{*}$ ) simultaneously. In other words, it is shown that one of the endemic equilibria must have a stable manifold in $\mathscr{D}^{*}$.

It is clear that since $\tilde{A}>0\left(\tilde{\mathscr{R}}_{0}<1\right)$, the equilibrium $e_{0}$ is locally asymptotically stable. Let $\Delta$ be the basin of attraction of $e_{0}$ and $\Omega=\Delta \cap \mathscr{D}^{*}$. Since $e_{0}$ attracts $\mathscr{D}_{0}$, it follows that $\mathscr{D}_{0} \subset \Omega$. Furthermore, since $\Delta$ is an open set, it can be seen that $\partial \Omega \cap \operatorname{int}\left(\mathscr{D}^{*}\right) \neq \varnothing$, where $\partial \Omega$ is the boundary of $\Omega$ and $\operatorname{int}\left(\mathscr{D}^{*}\right)$ is $\mathscr{D}^{*} \backslash \mathscr{D}_{0}$. Suppose $X^{0}=\left(S_{1}^{0}, V_{1}^{0}, I_{1}^{0}\right)$ is an arbitrary point in $\partial \Omega \cap \operatorname{int}\left(\mathscr{D}^{*}\right)$, which is not in the basin attraction of $e_{0}$. Let $\Phi\left(t, X^{0}\right)$ be a solution of the normalized model with $\Phi\left(0, X^{0}\right)=X^{0}$. Noting that $e_{1}$ and $e_{2}$ are located in the interior of $\mathscr{D}^{*}$, we can pick $X^{0}$ such that $X^{0} \notin\left\{e_{1}, e_{2}\right\}$. Since $X^{0}$ is not in the basin of attraction of $e_{0}$, it follows that $\Phi\left(t, X^{0}\right)$ cannot converge to $e_{0}$. Since $X^{0} \in \mathscr{D}^{*}$ and $\mathscr{D}^{*}$ is positively invariant, it follows that the $\omega$-limit set of the solution $\Phi\left(t, X^{0}\right)$ must be in $\mathscr{D}^{*} \backslash \Omega$. Furthermore, since the model has no periodic orbits in $\mathscr{D}^{*}$ (Theorem 3.3), the solution $\Phi\left(t, X^{0}\right)$ must converge to one of the two endemic equilibria. This implies that these equilibria cannot be both repellers in $\mathscr{D}^{*}$ simultaneously. Thus, although $e_{0}$ is locally asymptotically stable (since $\tilde{\mathscr{R}}_{0}<1$ ), this equilibrium point is not globally asymptotically stable. Thus, we have established the following theorem.

THEOREM 3.8. If $\tilde{A}>0, \tilde{B}^{2}-4\left(\mu^{2} / c^{2}\right) \tilde{\beta}_{1} \tilde{\beta}_{2} \tilde{A}>0$, and $\tilde{B}<0$, then the endemic equilibria of the normalized model cannot be repellers in $\mathscr{D}^{*}$ simultaneously. Furthermore, the equilibrium $e_{0}$ is not globally asymptotically stable.

The above result shows that one of the endemic equilibria has at least a onedimensional stable manifold. Without loss of generality, suppose this equilibrium is $e_{1}$. Further, suppose $e_{1}$ is in the interior of $\mathscr{D}^{*} \backslash \partial \Omega \cap \operatorname{int}\left(\mathscr{D}^{*}\right)$. Then, since the basin of attraction of $e_{0}$ is an open set (which does not include 
$\left.\partial \Omega \cap \operatorname{int}\left(\mathscr{D}^{*}\right)\right)$, discontinuity occurs in the direction field of the model in $\partial \Omega \cap$ $\operatorname{int}\left(\mathscr{D}^{*}\right)$. This is because solutions with initiating points very close to $X^{0}$, but in the basin of attraction of $e_{0}$, approach $e_{0}$. Consequently, $e_{1}$ must be located on $\partial \Omega \cap \operatorname{int}\left(\mathscr{D}^{*}\right)$ in the interior of $\mathscr{D}^{*}$. Therefore, the stable manifold of $e_{1}$ separates $\mathscr{D}^{*}$ into two basins of attraction. Furthermore, the unstable manifold of $e_{1}$ in the interior of $\mathscr{D}^{*} \backslash \partial \Omega \cap \operatorname{int}\left(\mathscr{D}^{*}\right)$ approaches $e_{2}$. This shows that the endemic equilibrium $e_{2}$ is stable. In summary, in this case, the model has two stable equilibria, namely, $e_{0}$ and $e_{2}$, and a saddle endemic equilibrium $e_{1}$ (where the stable manifold of $e_{1}$ separates the basins of attraction of $e_{0}$ and $e_{2}$ ).

4. The role of $\tilde{\mathscr{R}}_{0}$ on disease eradication. Since, for this model, the requirement $\tilde{\mathscr{R}}_{0}<1$ does not guarantee community-wide eradication of HIV, it is of public health interest to specifically determine the range of $\tilde{\mathscr{R}}_{0}$ that can ensure the global stability of $e_{0}$ (and, consequently, the community-wide eradication of HIV). In this section, the role of $\tilde{\mathscr{R}}_{0}$ in the global dynamics of $e_{0}$ is investigated. Consider $\tilde{\mathscr{R}}_{0}$ as a function of $\tilde{\xi}$ and let

$$
\tilde{R}_{1}=\frac{\tilde{\beta}_{1}}{1+\tilde{\tau}}, \quad \tilde{R}_{2}=\frac{\tilde{\beta}_{2}}{1+\tilde{\tau}} .
$$

Notice that since $\tilde{R}_{1} \geq \tilde{R}_{2}$ if $\tilde{R}_{2} \geq 1$, then

$$
\tilde{\mathscr{R}}_{0}(\tilde{\xi})=\tilde{R}_{2}+\frac{\tilde{R}_{1}-\tilde{R}_{2}}{1+\tilde{\xi}} \geq 1+\frac{\tilde{R}_{1}-\tilde{R}_{2}}{1+\tilde{\xi}} \geq 1
$$

In this case, no amount of vaccination is sufficient to bring $\tilde{\mathscr{R}}_{0}$ below 1 (which is a necessary condition for disease eradication). Therefore, from now on, we consider the case $\tilde{R}_{2}<1$. Suppose $\tilde{R}_{1}>1$. Differentiating $\tilde{\mathscr{R}}_{0}(\tilde{\xi})$ gives

$$
\tilde{\mathscr{R}}_{0}^{\prime}(\tilde{\xi})=\frac{\tilde{\beta}_{2}-\tilde{\beta}_{1}}{(1+\tilde{\xi})^{2}(1+\tilde{\tau})} .
$$

Since $\beta_{1} \geq \beta_{2}$ in the original model, it follows that $\tilde{\beta}_{1} \geq \tilde{\beta}_{2}$ in the normalized model. Thus, $\tilde{\mathscr{R}}_{0}(\tilde{\xi})$ is a decreasing function of $\tilde{\xi}$. It is easy to see that there is a unique critical vaccination rate $\tilde{\xi}_{c}=\left(1-\tilde{R}_{1}\right) /\left(\tilde{R}_{2}-1\right)$ such that $\tilde{\mathscr{R}}_{0}(\tilde{\xi}) \leq 1$ if $\tilde{\xi} \geq$ $\tilde{\xi}_{c}$ (with equality at $\tilde{\xi}=\tilde{\xi}_{c}$ ). We also note that $\tilde{\mathscr{R}}_{0}(0)=\tilde{R}_{1}$ and $\lim _{\tilde{\xi} \rightarrow \infty} \tilde{\mathscr{R}}_{0}(\tilde{\xi})=$ $R_{2}$. This implies that $\tilde{R}_{2} \leq \tilde{\mathscr{R}}_{0} \leq \tilde{R}_{1}$.

We now determine the range of $\tilde{\mathscr{R}}_{0}$ that guarantees disease eradication using Theorem 3.6. Note that $\tilde{B} \geq 0$ if and only if

$$
\tilde{\xi} \geq \frac{\tilde{\beta}_{2}\left(\tilde{R}_{1}-1\right)-\tilde{R}_{1}}{\tilde{R}_{2}}=\tilde{\xi}_{0} .
$$


It follows, using Theorem 3.6 (i), that $e_{0}$ is globally asymptotically stable whenever

$$
\tilde{\xi} \geq \max \left\{\tilde{\xi}_{c}, \tilde{\xi}_{0}\right\}=\tilde{\xi}_{*} .
$$

Thus, HIV will be eradicated from the community whenever $\tilde{R}_{2} \leq \tilde{\mathscr{R}}_{0} \leq \tilde{\mathscr{R}}_{0}\left(\tilde{\xi}_{*}\right)$.

We will extend our discussion by considering the quantity $\tilde{B}^{2}-4\left(\mu^{2} /\right.$ $\left.c^{2}\right) \tilde{\beta}_{1} \tilde{\beta}_{2} \tilde{A}$. Using the expressions for $\tilde{A}$ and $\tilde{B}$ (defined in (3.23)) gives

$$
\begin{aligned}
\tilde{B}^{2}-4 \frac{\mu^{2}}{c^{2}} \tilde{\beta}_{1} \tilde{\beta}_{2} \tilde{A}=\{ & \left(\tilde{\beta}_{2} \tilde{\xi}\right)^{2}+2 \tilde{\beta}_{2}\left[\tilde{\beta}_{1}+\left(\tilde{\beta}_{2}-2 \tilde{\beta}_{1}\right)(1+\tilde{\tau})+\tilde{\beta}_{1} \tilde{\beta}_{2}\right] \tilde{\xi} \\
& +\left[\tilde{\beta}_{1}+\tilde{\beta}_{2}(1+\tilde{\tau}+\tilde{d})-\tilde{\beta}_{1} \tilde{\beta}_{2}\right]^{2} \\
& \left.-4 \tilde{\beta}_{1} \tilde{\beta}_{2}\left[(1+\tilde{\tau})-\tilde{\beta}_{1}\right]\right\} \frac{\mu^{4}}{c^{2}} \\
\equiv & P(\tilde{\xi}) .
\end{aligned}
$$

Since $\tilde{R}_{1}>1$, it follows that

$$
\left[\tilde{\beta}_{1}+\tilde{\beta}_{2}(1+\tilde{\tau})-\tilde{\beta}_{1} \tilde{\beta}_{2}\right]^{2}-4 \tilde{\beta}_{1} \tilde{\beta}_{2}\left[(1+\tilde{\tau})-\tilde{\beta}_{1}\right]>0 .
$$

Thus, the quadratic $P(\tilde{\xi})$ either has no real root or has two real roots. If $P(\tilde{\xi})$ has real roots, then they must have the same sign (both negative or both positive). Notice that $P(0)>0$ and $\lim _{\tilde{\xi} \rightarrow \infty} P(\tilde{\xi})=\infty$. This implies that if $P(\tilde{\xi})$ has no real roots, then $P(\tilde{\xi})>0$ for $\tilde{\xi}>0$. Similarly, if $P(\tilde{\xi})$ has two negative real roots, then $P(\tilde{\xi})>0$ for $\tilde{\xi}>0$. Therefore, in order to establish the global stability of $e_{0}$ when $\tilde{B}^{2}-4\left(\mu^{2} / c^{2}\right) \tilde{\beta}_{1} \tilde{\beta}_{2} \tilde{A} \geq 0$, we require that $\tilde{\mathscr{R}}_{0}<1$ and $\tilde{B} \geq$ 0 . Thus, a similar argument to that of Theorem 3.6 (i) now shows that the conditions in Theorem 3.6 (ii) are always satisfied whenever $\tilde{\xi}>\tilde{\xi}_{*}$. This also implies that HIV will be eradicated from the community whenever $\tilde{R}_{2} \leq \tilde{\mathscr{R}}_{0} \leq$ $\tilde{\mathscr{R}}_{0}\left(\tilde{\xi}_{*}\right)$.

Finally, suppose $P(\tilde{\xi})$ has two positive real roots, namely, $\tilde{\xi}_{1}$ and $\tilde{\xi}_{2}$ with $\tilde{\xi}_{1}<\tilde{\xi}_{2}$. In this case, $\tilde{B}^{2}-4\left(\mu^{2} / c^{2}\right) \tilde{\beta}_{1} \tilde{\beta}_{2} \tilde{A}>0$ for $\tilde{\xi} \in\left[0, \tilde{\xi}_{1}\right) \cup\left(\tilde{\xi}_{2}, \infty\right)$ and $\tilde{B}^{2}-$ $4\left(\mu^{2} / c^{2}\right) \tilde{\beta}_{1} \tilde{\beta}_{2} \tilde{A} \leq 0$ for $\tilde{\xi} \in\left[\tilde{\xi}_{1}, \tilde{\xi}_{2}\right]$. However, as long as $\tilde{\xi}>\tilde{\xi}_{*}$, the conditions (i) and (ii) of Theorem 3.5 are satisfied. It should be noted that if $\tilde{R}_{1}<1$, then

$$
\tilde{\mathscr{R}}_{0}=\frac{\tilde{R}_{1}+\tilde{\xi} \tilde{R}_{2}}{1+\tilde{\xi}} \leq \tilde{R}_{1}<1,
$$

for any amount of vaccination (even $\tilde{\xi}=0$ ). It is easy to see that, in this case, the above discussion is valid. Hence, we have established the following theorem.

THEOREM 4.1. If $\tilde{R}_{2}<1$, then the equilibrium $e_{0}$ is globally asymptotically stable if $\tilde{\xi}>\tilde{\xi}_{*}$. If $\tilde{R}_{2} \geq 1$, then the unique endemic equilibrium is globally asymptotically stable and it attracts $\mathscr{D}^{*} \backslash \mathscr{D}_{0}$.

REMARK 4.2. It is easy to see that the same result can be obtained when $P(\tilde{\xi})$ has a real root of multiplicity $2\left(\tilde{\xi}_{1}=\tilde{\xi}_{2}\right)$. 
In summary, the epidemiological implication of the above theorem is that HIV will be eradicated from the community whenever $\tilde{\mathscr{R}}_{0} \leq \tilde{\mathscr{R}}_{0}\left(\tilde{\xi}_{*}\right)=\tilde{\mathscr{R}}_{*}$. It should be mentioned that $\tilde{\xi}_{*}$ (and consequently $\tilde{\mathscr{R}}_{*}$ ) exists as long as $\tilde{R}_{2}<1$. In other words, if $\tilde{R}_{2}>1$, no level of vaccination contributes to HIV eradication. It is clear that $\tilde{\mathscr{R}}_{*} \leq 1$ (when $\tilde{R}_{2}<1$ ) since $\tilde{\xi}_{*} \geq \tilde{\xi}_{c}$. If $\tilde{\mathscr{R}}_{*}<1$, then two endemic equilibria of the model exist as long as $\tilde{\mathscr{R}}_{*}<\tilde{\mathscr{R}}_{0}<1$. In this case, HIV eradication depends on the initial sizes of the three subpopulations of the model (in this scenario, HIV can only be eradicated if the initial sizes of the subpopulations are in the basin of attraction of $e_{0}$ ).

REMARK 4.3. The theoretical results of this paper confirm the possibility of bistability for some values of the vaccination parameter $\xi$ under some specific conditions. Here, we seek to explore the reason for the phenomenon of bistability in our model. To do so, we consider the case where $\xi=0$. In this case, the model reduces to the following two-dimensional vaccination-free (VF) model:

$$
\frac{d S}{d t}=\Pi-\frac{c \beta_{1} S I}{N}-\mu S, \quad \frac{d I}{d t}=\frac{c \beta_{1} S I}{N}-(\mu+\tau) I .
$$

Theoretical analysis of the above VF model reveals the following results.

(i) The VF model has a disease-free equilibrium $x_{0}=(\Pi / \mu, 0)$ which is locally asymptotically stable if $r_{0}<1$, where

$$
r_{0}=\frac{c \beta_{1}}{\mu+\tau}
$$

(ii) The model has a unique stable endemic equilibrium given by

$$
x_{1}=\left(\frac{\Pi}{c \beta_{1}-\tau}, \frac{\Pi\left(r_{0}-1\right)}{c \beta_{1}-\tau}\right)
$$

whenever $r_{0}>1$.

(iv) The positive quadrant $\Gamma=\{(S, I): S \geq 0, I \geq 0\}$ is a positively invariant region for the VF model.

(v) Using the Dulac function $D=1 / I$, it can be seen that the VF model has no periodic orbits in $\Gamma$.

(vi) The disease-free equilibrium $x_{0}$ is globally asymptotically stable if $r_{0} \leq$ 1 and unstable if $r_{0}>1$.

(vii) The endemic equilibrium $x_{1}$ is globally asymptotically stable if $r_{0}>1$.

Therefore, in the absence of vaccination $(\xi=0)$, the above results show that if $r_{0}<1$, HIV will be eradicated from the community. Thus, the VF model cannot exhibit bistability since no endemic equilibrium exists for $r_{0}<1$. We also note that in the presence of a perfect vaccine which offers $100 \%$ protection (i.e., $\beta_{2}=0$ ), the quadratic equation (3.9) reduces to a linear equation with at most one positive solution (corresponding to the unique endemic equilibrium). This implies that, if the vaccine is 100\% effective, the model (2.1), (2.2), 
and (2.3) cannot exhibit multiple endemic equilibria. These results strongly suggest that the low efficacy of vaccines (leading to $\beta_{2}>0$ ) is the reason for the bistability phenomenon in our three-dimensional-model (2.1), (2.2), and (2.3). The public health implication of this is that the use of vaccines with low efficacy will not guarantee community-wide eradication of HIV even when $\Re_{0}<1$.

5. Numerical simulations. In order to illustrate the theoretical results of the paper, the model was simulated under various scenarios (using Matlab software). In particular, we will monitor the effect of varying vaccination rates $\xi$ on the dynamical behaviour of the model. For simulation purposes, the following set of parameter values were used: $\Pi=50000$ per year, $\beta_{1}=0.06$ per contact, $\beta_{2}=0.006$ per contact, $\mu=0.02$ per year, $\tau=0.125$ per year and $c=10$ contact per year. The values of $\mu=0.02$ and $\tau=0.125$ represent a life expectancy of 50 years and a period of (approximately) 8 years, respectively, to progress to full-blown AIDS. With this choice of parameter values, the critical vaccination rate is $\xi_{c} \bumpeq 0.1025$ and $R_{2}=0.41<1$. Simulations were then run with varying values of $\xi$ (the vaccination rate) as follows.

5.1. Experiment 1: disease persistence. In this experiment, we chose $\xi=$ $0.09<\xi_{c}$ and initial condition $X_{0} \equiv(S(0), V(0), I(0))=(1000000,50000$, $1000)$. With this value of $\xi$, the basic reproductive number is $\mathscr{R}_{0}=1.07>1$. Simulation results obtained, tabulated in Table 5.1, show that the model has two equilibria: the disease-free equilibrium and a unique endemic equilibrium. The profile of $I(t)$, depicted in Figure 5.1, reveals that the solution with initial condition $X_{0}$ converges to the unique endemic equilibrium. This implies that the rate of vaccination $(\xi=0.09)$ is insufficient to eradicate HIV. Consequently, the disease persists within the community. These simulation results are consistent with the theoretical results given in Theorems 3.5 and 3.8.

5.2. Experiment 2: bistability. The goal of this experiment is to illustrate the coexistence of bistable equilibria of the model for some values of $\xi$. Here, we chose $\xi=0.105$ sufficiently small, but greater than $\xi_{c}=0.1025$. In this case, $\mathscr{R}_{0}=0.989<1$. Using an initial condition $X_{1}=(100000,5000,40)$, the profile of $I(t)$, depicted in Figure 5.2, shows that the solution with initial condition $X_{1}$ approaches the disease-free equilibrium $e_{0}$. On the other hand, the solution with initial condition $X_{2}=(100000,5000,100)$ approaches the stable endemic equilibrium $e_{2}$ (see Figure 5.2 and Table 5.1). These simulations reveal that the model exhibits bistability for this choice of $\xi$. In this case, community-wide eradication of HIV depends on the initial sizes of the subpopulations of the model.

It is easy to see, in this case, that $\xi_{0} \simeq 0.1086$ and consequently, $\xi_{*}=\xi_{0}$. Therefore, as predicted in Section 4, the model has bistable equilibria whenever $\xi_{c}<\xi<\xi_{*}$. It is worth mentioning that $\mathscr{R}_{0}\left(\xi_{*}\right) \simeq 0.973<\mathscr{R}_{0}=0.989<1$. 


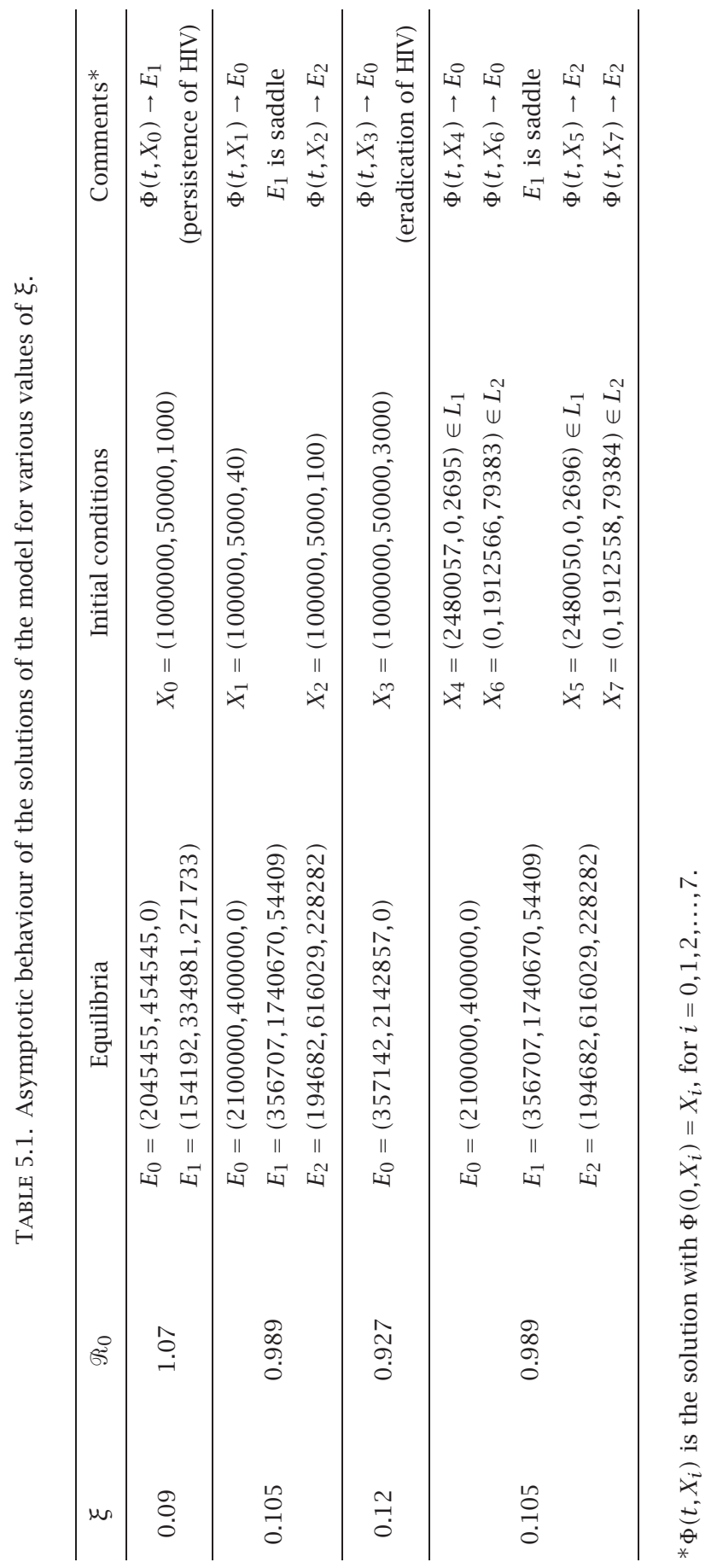




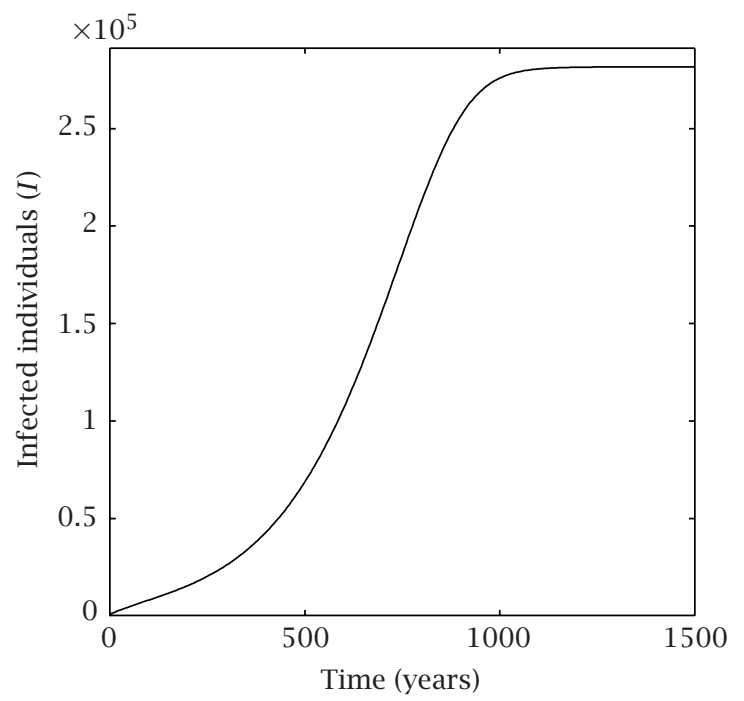

FIGURE 5.1. Profile of infected individuals $(I)$ for $\xi=0.09$ with initial condition $X_{0}$.

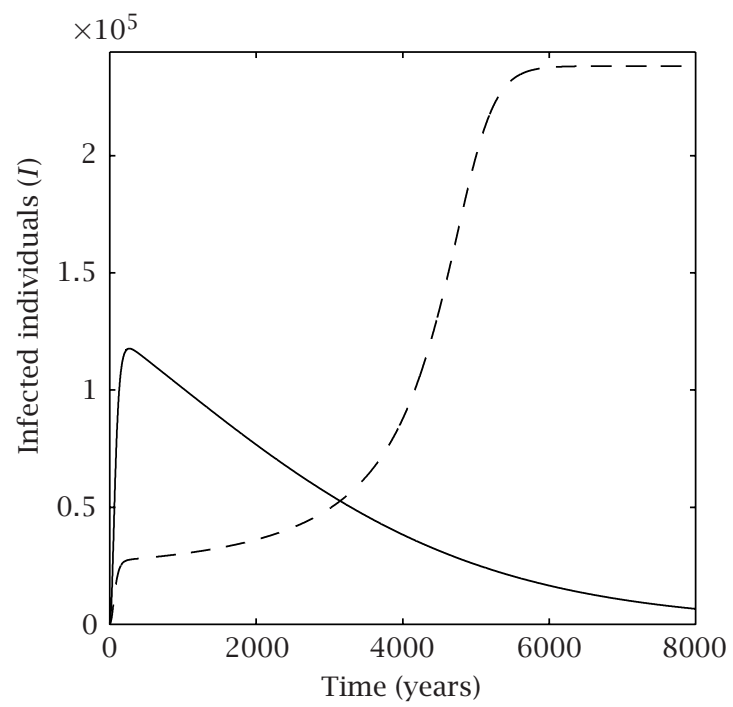

FiguRE 5.2. Profile of infected individuals $(I)$ for $\xi=0.105$ with two initial conditions $X_{1}$ and $X_{2}$. Solid line shows the profile of $10 \times I$ with initial condition $X_{1}$. Dashed line shows the profile of $I$ with initial condition $X_{2}$. 


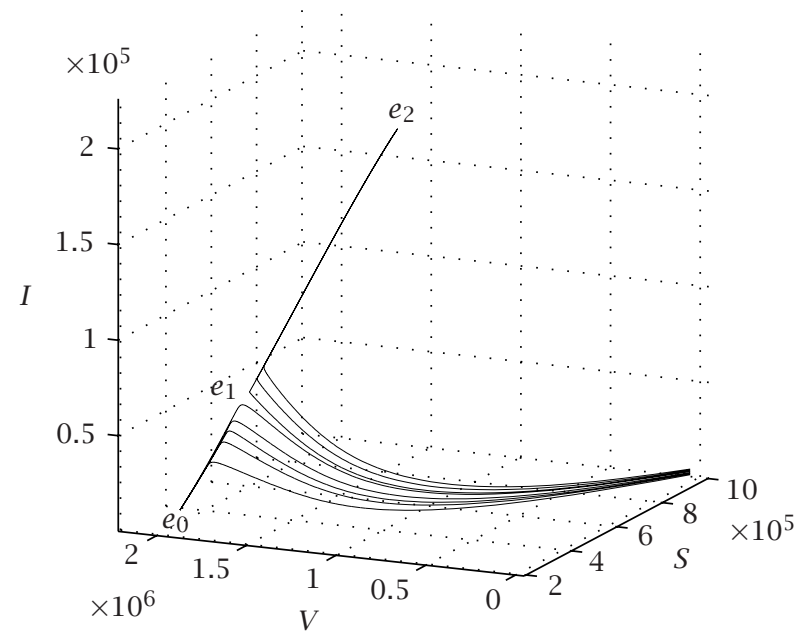

FIGURE 5.3. Phase diagram for $\xi=0.105$ with different initial conditions. This figure shows the coexistence of stable disease-free equilibrium $\left(e_{0}\right)$ with the stable endemic equilibrium $\left(e_{2}\right)$. The stable manifold of the saddle point $e_{1}$ separates the basins of attraction of $\left(e_{0}\right)$ and $\left(e_{2}\right)$.

The above simulations demonstrate that the disease-free equilibrium and one of the two endemic equilibria (namely, $e_{2}$ ) are locally asymptotically stable whenever $\xi_{c}<\xi<\xi_{*}$ (see Figure 5.3 and Table 5.1). Noting that $\mathscr{D}^{*}$ is a positively invariant region for the normalized model, we may restrict our attention to the basins of attraction of the stable equilibria in $\mathscr{D}^{*}$. Since the basin of attraction of an attractor is an open set (by definition), it follows that $\mathscr{D}^{*}$ is separated by the stable manifold of the saddle point $\left(e_{1}\right)$ into the basins of attraction of these two attractors $\left(e_{0}\right.$ and $\left.e_{2}\right)$. These basins can be numerically specified by finding the points where the stable manifold of the saddle point intersects the boundary of two-dimensional simplex $\mathscr{D}^{*}$. This intersection consists of exactly two points at which the $\omega$-limit set of the solutions of the model, with initial conditions on the boundary of $\mathscr{D}^{*}$, changes from one attractor to another.

For example, in the case where $\xi=0.105$, this intersection consists of two points $P_{1}$ and $P_{2}$ where $P_{1}$ is located on line $L_{1}=\{(S, 0, I): 0.02 S+0.148 I=$ $50000\}$ and $P_{2}$ is located on line $L_{2}=\{(0, V, I): 0.02 V+0.148 I=50000\}$ in the original model coordinates. Numerical results indicate that every solution of the model with the initial condition on the line $L_{1}$ approaches $e_{0}$ if $I \leq 2695$ and it approaches $e_{2}$ if $I \geq 2696$. Further simulations also reveal that every solution with the initial condition on the line $L_{2}$ approaches $e_{0}$ if $I \leq 79383$ and approaches $e_{2}$ if $I \geq 79384$. 


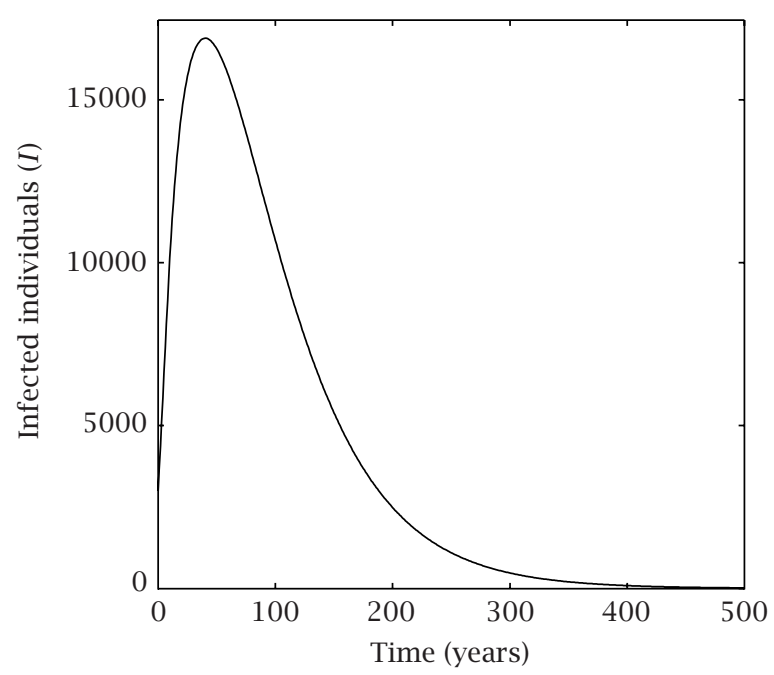

FIGURE 5.4. Profile of infected individuals ( $I$ ) for $\xi=0.12$ with initial condition $X_{3}$.

5.3. Experiment 3: disease eradication. In this experiment, we chose $\xi=$ $0.12>\xi_{*}=0.1085$ and initial condition $X_{3}=(1000000,50000,3000)$. With this vaccination rate, the basic reproductive number is $\mathscr{R}_{0}=0.927<\mathscr{R}_{*}=$ 0.973 . Notice that this vaccination rate is slightly greater than $\xi_{*}$. In this case, the model has only the disease-free equilibrium $e_{0}$ (see Table 5.1). Simulation results, depicted in Figure 5.4, show that HIV will be eradicated from the community. This experiment is also consistent with Theorem 4.1.

6. Discussion. In this paper, we proposed and qualitatively analyzed a deterministic model for HIV epidemiology that incorporates an anti-HIV preventive vaccine. The local stability of the disease-free equilibrium was established, based on a certain threshold quantity known as the basic reproductive number.

Although the endemic equilibria of the model cannot be clearly expressed in a closed form, the existence of endemic equilibria was established under some specific conditions by finding the fixed point of the equation for the force of infection [17]. Using the technique proposed in [2], we proved the nonexistence of certain types of solutions such as periodic orbits, homoclinic orbits, or polygons for the normalized model. This enabled us to establish the local and global stability of the model. The results of the global analysis of the model allowed the determination of a threshold vaccination rate $\xi_{*}$, leading to disease eradication if $\xi>\xi_{*}$. This threshold quantity $\left(\xi_{*}\right)$ gives a subthreshold domain of bistable equilibria of the model, namely, $\mathscr{R}_{*}=\mathscr{R}_{0}\left(\xi_{*}\right)<\mathscr{R}_{0}<1$.

Our study shows that, like models of some curable diseases (see [9, 13] and the references therein), our HIV model can also exhibit bistable equilibria (involving the disease-free equilibrium and an endemic equilibrium) whenever 
$\mathscr{R}_{*}<\mathscr{R}_{0}<1$. In this case, the initial sizes of the subpopulations determine which of the two stable equilibria is reached. Thus, controlling the sizes of the model subpopulations can lead to HIV eradication in place of persistence. By analyzing the VF model, it was also shown that the low efficacy of vaccine is the reason for the bistability in our model.

In summary, the results of this study show that if the efficacy of vaccine is not high enough (leading to $R_{2}>1$ ), no amount of vaccination rate could lead to HIV eradication. However, if the vaccine efficacy can reduce the probability of infection such that $R_{2}<1$ (i.e., $\beta_{2}$ is low enough), increasing the rate of vaccination to $\xi>\xi_{*}$ guarantees HIV eradication.

ACKNOWLEDGment. This work was supported in part by the Natural Sciences and Engineering Research Council of Canada (NSERC). The authors are grateful to the referees for their comments which have significantly enhanced the paper.

\section{REFERENCES}

[1] R. M. Anderson and R. M. May, Infectious Diseases of Humans, Oxford University Press, London, 1991.

[2] S. Busenberg and P. van den Driessche, Analysis of a disease transmission model in a population with varying size, J. Math. Biol. 28 (1990), no. 3, 257-270.

[3] C. Castillo-Chavez, Z. Feng, and W. Huang, On the computation of $\mathscr{R}_{0}$ and its role on global stability, Mathematical Approaches for Emerging and Reemerging Infectious Diseases: An Introduction (Minneapolis, Minn, 1999), IMA Vol. Math. Appl., vol. 125, Springer, New York, 2002, pp. 229-250.

[4] J. Dushoff, W. Huang, and C. Castillo-Chavez, Backwards bifurcations and catastrophe in simple models of fatal diseases, J. Math. Biol. 36 (1998), no. 3, 227-248.

[5] M. Fan, M. Y. Li, and K. Wang, Global stability of an SEIS epidemic model with recruitment and a varying total population size, Math. Biosci. 170 (2001), no. 2, 199-208.

[6] Z. Feng, C. Castillo-Chavez, and A. F. Capurro, A model for tuberculosis with exogenous reinfection, Theoret. Population Biol. 57 (2000), no. 3, 235-247.

[7] D. Greenhalgh, O. Diekmann, and M. C. M. de Jong, Subcritical endemic steady states in mathematical models for animal infections with incomplete immunity, Math. Biosci. 165 (2000), no. 1, 1-25.

[8] D. Greenhalgh, M. Doyle, and F. Lewis, A mathematical treatment of AIDS and condom use, IMA J. Math. Appl. Med. Biol. 18 (2001), no. 3, 225-262.

[9] A. B. Gumel and S. M. Moghadas, A qualitative study of a vaccination model with non-linear incidence, Appl. Math. Comput. 143 (2003), no. 2-3, 409-419.

[10] K. P. Hadeler and C. Castillo-Chavez, A core group model for disease transmission, Math. Biosci. 128 (1995), no. 1-2, 41-55.

[11] K. P. Hadeler and P. van den Driessche, Backward bifurcation in epidemic control, Math. Biosci. 146 (1997), no. 1, 15-35.

[12] Y.-H. Hsieh and S.-P. Sheu, The effect of density-dependent treatment and behavior change on the dynamics of HIV transmission, J. Math. Biol. 43 (2001), no. 1, 69-80. 
[13] C. M. Kribs-Zaleta, Center manifolds and normal forms in epidemic models, Mathematical Approaches for Emerging and Reemerging Infectious Diseases: An Introduction (Minneapolis, Minn, 1999), IMA Vol. Math. Appl., vol. 125, Springer, New York, 2002, pp. 269-286.

[14] C. M. Kribs-Zaleta and J. X. Velasco-Hernández, A simple vaccination model with multiple endemic states, Math. Biosci. 164 (2000), no. 2, 183-201.

[15] M. Y. Li, H. L. Smith, and L. Wang, Global dynamics of an SEIR epidemic model with vertical transmission, SIAM J. Appl. Math. 62 (2001), no. 1, 58-69.

[16] S. M. Moghadas and A. B. Gumel, Global stability of a two-stage epidemic model with generalized non-linear incidence, Math. Comput. Simulation 60 (2002), no. 1-2, 107-118.

[17] J. X. Velasco-Hernández, A model for Chagas disease involving transmission by vectors and blood transfusion, Theoret. Population Biol. 46 (1994), no. 1, $1-31$.

[18] J. X. Velasco-Hernández and Y.-H. Hsieh, Modelling the effect of treatment and behavioral change in HIV transmission dynamics, J. Math. Biol. 32 (1994), no. 3, 233-249.

B. D. Corbett: Department of Mathematics, University of Manitoba, Winnipeg, Manitoba, Canada R3T 2N2

E-mail address: umcorbe1@cc.umani toba.ca

S. M. Moghadas: Department of Mathematics, University of Manitoba, Winnipeg, Manitoba, Canada R3T 2N2

E-mail address: seyed.moghadas@nrc-cnrc.gc.ca

A. B. Gumel: Department of Mathematics, University of Manitoba, Winnipeg, Manitoba, Canada R3T 2N2

E-mail address: gume1ab@cc. umani toba.ca 


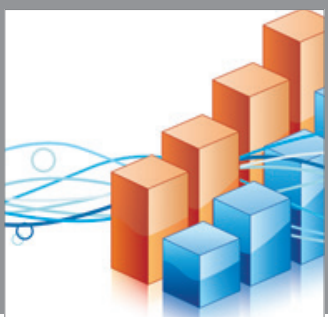

Advances in

Operations Research

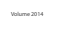

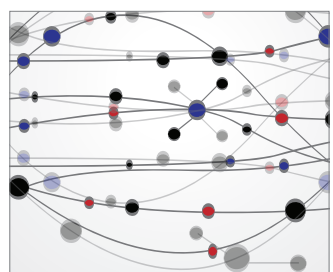

\section{The Scientific} World Journal
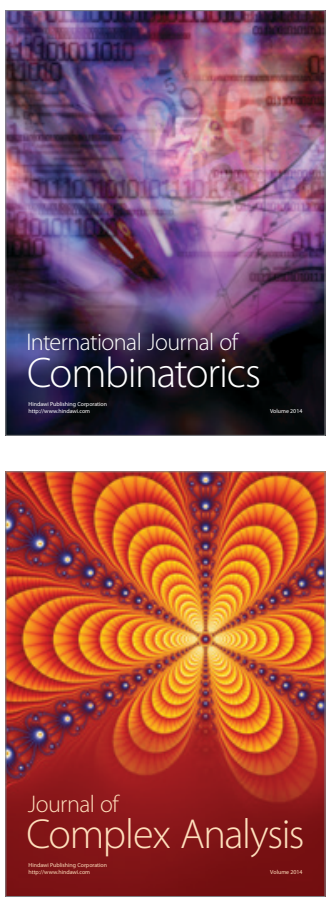

International Journal of

Mathematics and

Mathematical

Sciences
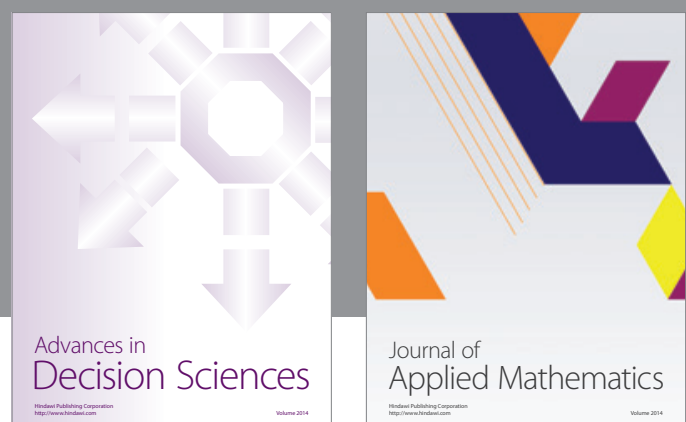

Journal of

Applied Mathematics
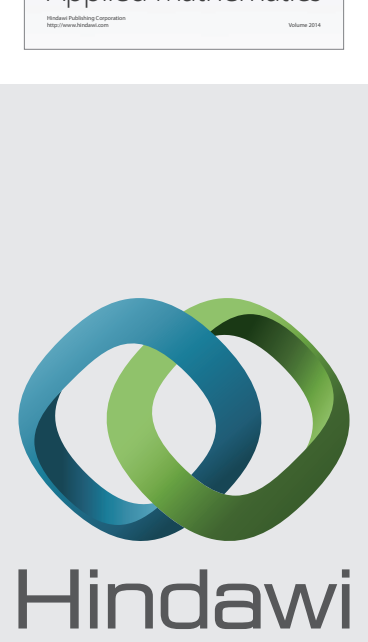

Submit your manuscripts at http://www.hindawi.com
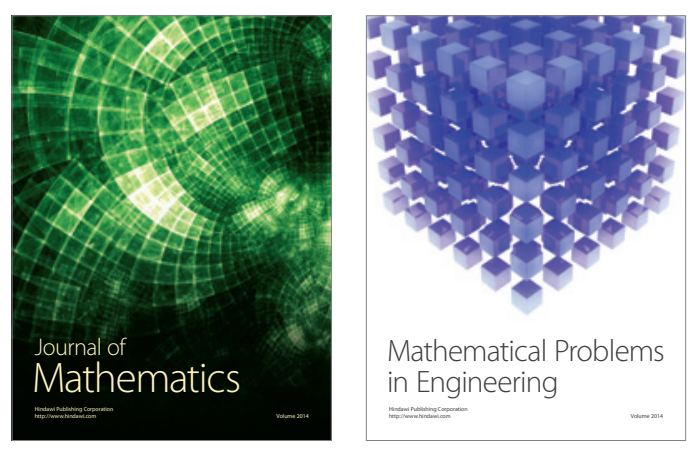

Mathematical Problems in Engineering
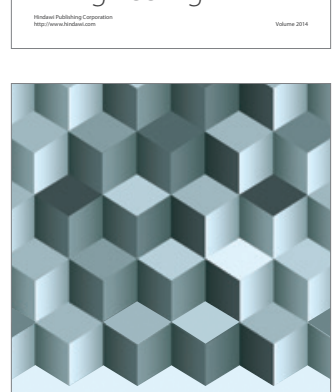

Journal of

Function Spaces
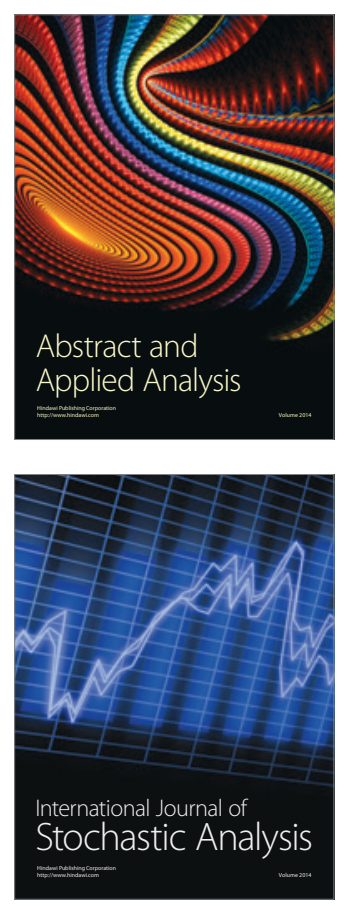

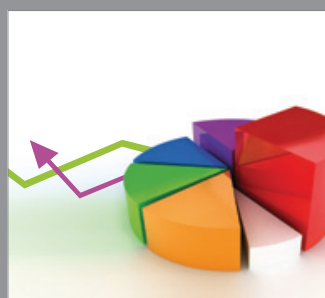

ournal of

Probability and Statistics

Promensencen
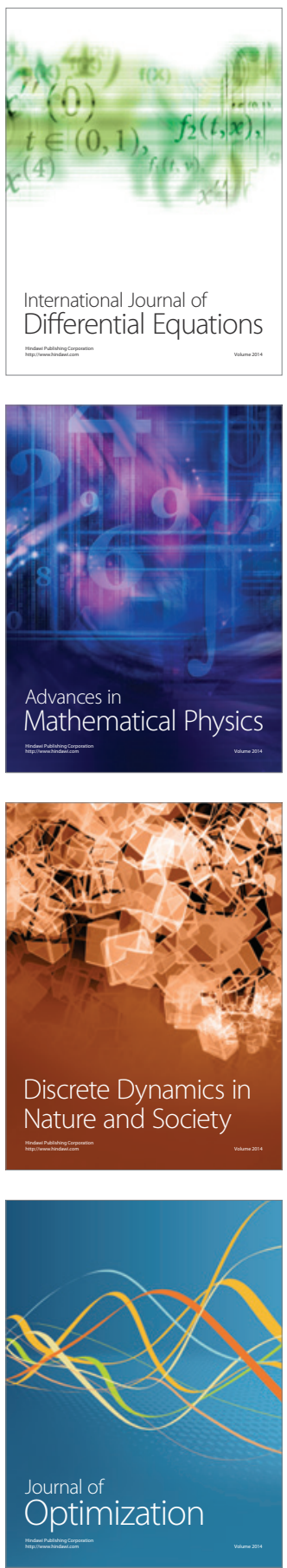